\title{
Carotid Artery Dissection
}

National Cancer Institute

\section{Source}

National Cancer Institute. Carotid Artery Dissection. NCI Thesaurus. Code C125662.

Spontaneous or traumatic separation of the layers of the carotid artery wall. It manifests with headache, neck pain, temporary vision loss, and/or ischemic stroke. 